\title{
Origin of Structures in Wolf-Rayet Winds: FUSE Observations of WR $135^{1}$
}

\author{
S.V. Marchenko ${ }^{2}$, A.F.J. Moffat ${ }^{3}$, N. St-Louis ${ }^{3}$, A.W. Fullerton ${ }^{4,5}$
}

\begin{abstract}
We report the detection with FUSE of strong, highly blue-shifted absorption features appearing in the absorption troughs of practically all major $\mathrm{P}$ Cygni profiles in the presumably single Wolf-Rayet star WR135. These features also appear in the shock-sensitive OVI 1032/38 A doublet, coincident both in time and velocity space with the rest of the lower-ionization species. Choosing between two alternative interpretations: large-scale, coherent structures vs. localized, random shocks, we favor the latter. The absolute value of velocity, as well as velocity dispersion in the shocked region, the density of the shocked gas, and the time scales of the observed variability allow us to relate the observed shocks to the incidence of numerous over-dense clumps (blobs) in the wind of a hot, massive star.
\end{abstract}

Subject headings: stars: winds, outflows — stars: activity — stars: Wolf-Rayet — stars: individual (WR 135)

\section{Introduction}

All massive $\left(M_{\text {init }} \sim 20-100 M_{\odot}\right)$ stars drive strong winds. These stars spend most of their lifetimes in the hot part of the HRD, either as O (H-burning) or Wolf-Rayet (WR:

\footnotetext{
${ }^{1}$ Based on observations made with the NASA-CNES-CSA Far Ultraviolet Spectroscopic Explorer. FUSE is operated for NASA by The Johns Hopkins University under NASA contract NAS5-32985.

${ }^{2}$ Department of Physics and Astronomy, Western Kentucky University, 1906 College Heights Blvd., 11077, Bowling Green, KY 42101-1077, USA; sergey.marchenko@wku.edu

${ }^{3}$ Département de Physique and Observatoire du Mont Mégantic, Université de Montréal, CP 6128, Succursale Centre-Ville, Montréal, QC H3C 3J7, moffat,stlouis@astro.umontreal.ca

${ }^{4}$ Department of Physics and Astronomy, University of Victoria, P.O. Box 3055, Victoria, BC, Canada.

${ }^{5}$ Center for Astrophysical Sciences, Department of Physics and Astronomy, The Johns Hopkins University, 3400 North Charles Street, Baltimore, MD 21286, USA; awf@pha.jhu.edu
} 
mainly He-burning) stars. Although hot-star winds were suspected to be inhomogeneous (e.g. Cherepashchuk et al. 1984), truly direct evidence came only from the detection of stochastic, systematically moving subpeaks on the strong emission lines of WR stars (Moffat et al. 1988). This profoundly changed the approach in atmospheric modeling of WR stars (e.g. Hillier 1991; Hamann \& Koesterke 1998, Hillier \& Miller 1999) and resulted in a substantial reduction, by a factor 2-5, of predicted mass-loss rates. A similar factor may also apply to O-star winds (e.g. Eversberg et al. 1998, Donati et al. 2002, Fullerton, Massa \& Prinja 2005). Since the evolution of massive stars is heavily affected by mass-loss, such a significant reduction in mass-loss rate called for a major revision in models of massive stars and stimulated further research (see Meynet \& Maeder 2005, and references therein).

Although the consequences of wind inhomogeneities for mass-loss estimates are understood, the origin of the clumps is not. It is almost certain that radiative instabilities (e.g. Owocki 1994) must ultimately lead to supersonic, compressible turbulence (Henriksen 1994). Then it is reasonable to assume that the resulting shocks will generate the X-ray fluxes seen emanating (e.g. Berghoefer et al. 1997, Wessolowski 1996) from all hot-star winds. Linking shocks to X-rays, one should look for a stochastic variablility of X-ray flux, expecting that rather infrequent shock-shock collisions are the most influential events in X-ray production (Feldmeier et al. 1997). However, no X-ray facility is sensitive enough yet to detect such fluctuations (Berghöfer et al. 1997). In addition, the situation is complicated by the large optical depth of the outflows from WR stars. While the clumps are routinely observed at the base of the wind $r_{\sim}^{<100} R_{\star}$ where the emission lines arise, the [presumably related] X-ray flux emerges from $r \gg 100 R_{\star}$.

In order to establish that embedded shocks are responsible for the clumpy structure of hot-star winds, one may rely on observations of resonance lines of "super-ions," i.e., ions whose presence cannot be explained by photoionization in winds characterized by temperatures between 20 and $40 \mathrm{kK}$ (e.g. MacFarlane et al. 1994). The best superionization feature is the strong OVI resonance line with $\chi_{\text {ioniz }}=114-138 \mathrm{eV}$ at $1031.93 / 37.62 \AA$, i.e. accessible only with Far Ultraviolet Spectroscopic Explorer (FUSE). If the OVI line feature is formed in hot, shocked gas filaments (clumps) permeating the accelerating wind, one should see a variable profile, with corresponding statistical properties (velocity dispersion, timescales, amplitudes) resembling those found in optical emission lines, presumably produced via superposition of counltless clumps (Lépine \& Moffat 1999). One may also expect, by analogy with the optical lines, the non-saturated P Cygni absorption parts of the profiles to show the highest level of variability.

Our target, the presumably single, luminous Wolf-Rayet star WR135= HD 192103 (WC8) is known to show a highly variable, structured (clumpy) wind (Lépine et al. 2000), 
as well as being an X-ray source (ROSAT: $2.1 \pm 1.7\left[10^{-3} c t s s^{-1}\right]$, although barely exceeding a $1 \sigma$ detection limit - see arguments in Oskinova et al. 2003). In this star, as with virtually all other WR stars observed in the optical (Lépine \& Moffat 1999), spectral subpeaks are seen to propagate outwards towards the edges of strong emission lines. These peaks are interpreted as density enhancements emitting excess recombination-line radiation, propagating on average along with the general wind outflow. In WR 135 the moving subpeaks typically range up to $0.2-2.0 \%$ of the underlying emission-line strength, have a radial velocity dispersion $\sigma_{r}=$ $175 \mathrm{~km} \mathrm{~s}^{-1}$, and last for 7.5 hours on average (Lépine et al. 2000). The spectrum of WR135, leaving aside the prominent O VI feature, can be modeled with $T_{\text {eff }}\left(\tau_{\text {Ross }}=2 / 3\right)=2.710^{4}$ $\mathrm{K}$ (Dessart et al. 2000). On the other hand, the O VI doublet, the central subject of our discussion, peaks in strength at $T \sim 310^{5} \mathrm{~K}$ (Zsargó et al. 2003, Sembach et al. 2004). This high ionization, two stages above the dominant level (Cassinelli \& Olson 1979), is explained by Auger ionization in the presence of X-rays generated in numerous wind-embedded shocks.

Here we report the simultaneous appearence of short-lived (hours) absorption features in the high-velocity, blueshifted flanks of all major P Cygni emission profiles (OVI included) in the FUSE range. We link these absorption features to strong, highly supersonic shocks embedded in the wind of WR 135.

\section{FUSE Observations}

We observed WR 135 with FUSE (Moos et al. 2000, Sahnow et al. 2000) in October 2004, starting at HJD 2453287.5930 (UT=02:11, Oct. 9), and ending at HJD 2453288.8483 (UT 08:19, Oct. 10), thus following the star for 30+ hours practically non-stop. The spectra were obtained in histogram (HIST) mode, with the MDRS $(4 " \times 20 ")$ aperture, covering 910-1185 A . Initially, 59 exposures (root name D0440101) were scheduled back-to-back over 18 consecutive orbits. However, for technical reasons, only 42 spectra were obtained, with a particularly large, $\sim 4$ h gap during planned exposures 15-29, when detectors autonomously shut down. Exposures 5 and 55 were skipped because of acquisition problems. The majority of exposures were taken with $T_{\text {exp }} \sim 500 \mathrm{~s}$. The main setback came from a mis-alignment of the $\mathrm{SiC}$ channels causing loss of flux. However, the LiF channels performed well. The latest version of CalFUSE (v3.0.7) was used to extract and calibrate spectra from the two LiF channels.

Armed with a large number of spectra of the same object obtained within a short time interval, we tried to maximize the signal-to-noise ratio $(\mathrm{S} / \mathrm{N})$ of individual exposures. The presence of strong, frequently saturated IS absorptions called for minimal usage of any binning in velocity space on the original spectra. However, it did not preclude an appropriate 
smoothing of the differences (individual minus average, individual minus individual). We assembled the spectra into groups, combining together the $\mathrm{A}$ and $\mathrm{B}$ segments of the $2 \mathrm{LiF}$ detectors into 4 separate averages and then inter-comparing the averages. Before combining the spectra, we corrected all small-scale deviations $\left(<3 \mathrm{~km} \mathrm{~s}^{-1}\right)$ in the rest-frame radial velocities of strong interstellar absorption lines. By comparing the averages, we found that the LiF2A and LiF1B segments have practically the same $\mathrm{S} / \mathrm{N}$, although the LiF1B flux was reduced by $10 \%$. On the other hand, $\mathrm{LiF} 2 \mathrm{~B}$ has a $\mathrm{S} / \mathrm{N}$ that is two times lower than that of LiF1A. Hence, initially we treated all 4 segments separately, then appropriately combined the processed $\mathrm{LiF} 2 \mathrm{~A}$ with $\mathrm{LiF} 1 \mathrm{~B}$ and $\mathrm{LiF} 2 \mathrm{~B}$ with $\mathrm{LiF} 1 \mathrm{~A}$ at the very end of the procedure. This also provided an efficient line-by-line cross-check of the suspected variability patterns. Experimenting with various grouping algorithms, we finally decided to average all available spectra with comparable $\mathrm{S} / \mathrm{N}$ levels, taking the median values and additionally rejecting at each given wavelength the 10 most deviating flux readings (5 maximal and 5 minimal). Then the average was subtracted from each individual spectrum, channel-by-channel, and the difference was smoothed with a Gaussian filter. Subtracting the smoothed difference from the former (thus removing the low-frequency instrumental trends but retaining the high-frequency signal), and then subtracting this second-order difference from the original spectrum (thus reducing the high-frequency noise), we managed to increase the $\mathrm{S} / \mathrm{N}$ in each individual spectrum from the initial $\sim 15$ to $\sim 40$, without degrading the spectral resolution. Finally, by carefully assessing the spectrum-to-spectrum variability, we found that the processed spectra can be assembled into 3 groups (Fig. 1): exposures 1-14 (midtime HJD 2453287.6664), 30-39 (HJD 2453288.1803) and 40-59 (HJD 2453288.6066).

\section{Results and Discussion}

Despite the overall improvement in S/N, our detection limits do not exceed 5-10\% (refering to the ambient continuum), depending on the spectral range, in any individual exposure. Fixed-pattern noise, i.e. small-scale and fairly stable, within a few hours, noise patterns are mainly responsible for the problem. These detection limits are $\sim$ a factor of two lower in the grouped exposures. Within these limits the emission parts of all major transitions can be considered as constant. Recall that in the optical lines the variability does not exceed $\sim 2 \%$ (Lépine et al. 2000). In Fig. 1 we show the major variable part that of the absorption troughs of the P Cygni profiles. To normalize the velocities, we used the terminal wind velocity $v_{\infty}=1479 \mathrm{~km} \mathrm{~s}^{-1}$ from Willis et al. (2004), which fits the data better than $v_{\infty}=1343 \pm 496 \mathrm{~km} \mathrm{~s}^{-1}$ from Niedzielski \& Skorzynski (2002).

Finally we converted the detected variations to the differences of apparent optical depths, 
$d=\ln (F 1 / F 2)$ (Savage \& Sembach 1991), where F1 is an average of exposures 1-14, and F2 is either an average of exposures 30-39 or an average of exposures 40-59 (see Fig.1). To reduce the noise, we removed all artefacts introduced by the saturated features (literally, the results of $0 / 0$ division) and smoothed the ratio $\mathrm{F} 1 / \mathrm{F} 2$ using a gaussian filter with $\mathrm{FWHM}=15$ pix. The smoothing does not change the original FWHM values of the features, however slightly $(\underset{\sim}{<10 \%)}$ reduces their depths. One immediately notices (Fig. 2, exposures 1-14 vs. exposures 40-59) the high-velocity, $v \approx-1.5 \rightarrow-1.0 v_{\infty}$ feature seen in all well-developed $\mathrm{P}$ Cygni profiles across the spectrum. The excellent match between these features seen in both components of the S IV doublet lends additional credibility to the result. This absorption component appears to be much wider in the CIII line, which can be explained by the multiplet nature of this transition, with contributing components being separated by as much as $\sim 0.1 v_{\infty}$. While preparing Fig. 2, we tentatively assigned $\lambda_{0}=1175.7 \AA$ to $\mathrm{C}$ III, noticing that the components are spread between $\lambda \lambda=1174.93-1176.37 \AA$. Another prominent feature at $v \approx-0.8 \rightarrow-0.2 v_{\infty}$ is seriously affected by saturation (OVI, S IV and CIII profiles), which causes the displacements, or even near disappearance of this feature. However, this absorption component is clearly seen in the non-saturated Si III profile. From now on we focus our attention on the feature less affected by saturation at $v \approx-1.5 \rightarrow$ $-1.0 v_{\infty}$, which, being above $v_{\infty}$, is practically free from the ambiguity introduced by the underlying, forward-scattering emission component.

We notice the symmetric appearance of this blueshifted absorption in S IV, as expected for a feature originating in an optically thin region with radially isotropic (backward/forward) turbulence. The asymmetry, the blueshift and the larger extension of the CIII ( and, to a lesser extent, O VI) feature can be related to the high optical depth (i.e., substantial selfabsorption) of the transition and saturation of the relevant part of the profile: compare $\mathrm{S}$ IV or O VI to CIII at $v_{\sim}^{>}-1.15 v_{\infty}$ in Fig. 1.

Apparently, the velocity spacing of the OVI doublet places the variable $\left(v<-1.2 v_{\infty}\right)$ part of the absorption trough of the $1037.6 \AA$ component (Fig. 1) on the $v<-0.1 v_{\infty}$ emission part of the $1031.9 \AA$ line, which may complicate the interpretation, if one assumes that the observed variability could be caused by changes in the underlying emission. For the OVI $1037.6 \AA$ transition the detection of variability at $v<-0.2 v_{\infty}$ is impaired by the presence of strong, saturated IS absorptions. However, we remedy with the CIII $1175.7 \AA$ complex. Its strong, well-defined variability at $v<-1.2 v_{\infty}$ (Fig. 1) is not accompanied by any detectable changes in the $-0.4 v_{\infty}<v<-0.1 v_{\infty}$ part of the profile (Fig.2).

There are two possible explanations for the detected blue-edge $\left(v>v_{\infty}\right)$ variability: discrete absorption components (DACs) and fairly localized, wind-embedded shocks. The DACs are thought to be related to the co-rotating interaction regions (CIRs: Cranmer \& 
Owocki 1996), the zones where high-speed streams generated by disturbances at the base of the wind collide with relatively slower parts of the wind. One should notice the high incidence of DACs in the winds of OB stars (Kaper et al. 1996) vs. relative rarity of these large-scale, spatially-coherent structures in the WR winds (see Marchenko 2003 and references therein).

Have we captured a single, isolated, stochastically appearing shock or a CIR-like structure which periodically crosses the line of sight? We favor the former, as there is no trace of any large-scale, coherent structures in the optical (Lépine et al. 2000). Plus, the wind of WR135 is not flattened, contrary to all the CIR-bearing examples: WR6, WR134 and WR137 (see Lefèvre et al. 2005 and references therein). In OB stars any CIR-related variability takes place in a wide velocity range, $-v_{\infty}<v<v_{\infty}$, practically never exceeding $v_{\infty}$ (rather, slightly modulating the blueshifted absorption edge: Kaper \& Henrichs 1994). In WR135 we see a clear dominance of variability which goes far beyond $-v_{\infty}$, by as much as $50 \%$. And, we see no signs of variability exceeding the $5-10 \%$ detectability levels in the 'traditional' CIR domain, $-v_{\infty}<v<v_{\infty}$ (cf. the well-studied case of WR6: St-Louis et al. 1995). In WR135, rather than modulating the $-v_{\infty}<v$ edge, as usually attributable to CIRs, the short-living additional absorption is perceived as an isolated feature (cf. S IV 1073 in Fig. 1) with no signs of a gradual shift to higher velocities (cf. CIII 1175.7 in Fig. 1). In addition, the timescales of the detected UV variability, $\sim 0.5-1.0 \mathrm{~d}$, correspond reasonably well to the average 'survival' times of blobs in the optical region, $\underset{\sim}{<} .5 \mathrm{~d}$ (Lépine et al. 2000). And, the FWHMs of the features seen in Fig. $2, F W H M \approx 400 \mathrm{~km} \mathrm{~s}^{-1} \approx 2.4 \sigma$ (assuming a Gaussian profile) closely match the observed value of the radial velocity dispersion in the blobs seen in optical emission lines, $\sigma=175 \mathrm{~km} \mathrm{~s}^{-1}$. The synchronized appearence of the absorption features in all major P Cygni profiles across the FUSE spectrum mimics the general correlation in the behavior of blobs observed in different lines in the optical (Lépine et al. 2000, Lefèvre et al. 2005). Hence, one may relate the observed traces of shocked (and rapidly cooling, thus getting denser) gas to the incidence of numerous over-dense clumps (blobs) in the wind of WR135. Of course, the great spatial coherence of a DAC-related structure may facilitate detection. On the other hand, while adopting the model of a structured (clumpy) WR wind, one must count on the presence of a large amount of profile-forming, overdense structures (e.g. Lepine et al. 2000). The sheer number of stochastically distributed inhomogeneities leads to high probability of seeing, at any given moment, such a structure in absorption (i.e., projected on the optically thick part of the wind).

One may provide additional arguments favoring the 'shock' scenario over DAC (CIR)related variability. There are numerous examples of $v>v_{\infty}$ variability in WR stars observed by IUE: WR 136 (St-Louis et al. 1989), WR 6 (St-Louis et al. 1995), to name a few. An extensive search for OVI variability in the winds of OB stars (Lehner et al. 2003) shows that a solid majority, at least $64 \%$ of the O3-B1 targets, can be considered as variable. However, 
none of the surveyed objects showed $v>v_{\infty}$ variations in O VI, despite the tendency of stronger shocks to occur more frequently near $v_{\infty}$, and despite the tendency of more than $80 \%$ of O stars (Howarth \& Prinja 1989) to show DACs in their spectra. Hence, the O VI line seems to be less sensitive to the DAC-related phenomena in comparison to other strong UV transitions. The only notable exception is $\alpha \mathrm{Cam}$, where the detected variability in UV resonance lines (Lamers et al. 1988) extends far beyond the derived $v_{\infty}=1550 \mathrm{~km} \mathrm{~s}^{-1}$ (Repolust et al. 2004).

If we are to interpret the variability as some kind of relaxation of the initially 'unperturbed' profile (red line in Fig. 1), then the OVI profile has the highest restoration pace. This could be related to fast cooling of the shocked gas. Also, note that in the CIII and SIV profiles the higher-velocity (thus presumably higher-temperature) region is restored much faster.

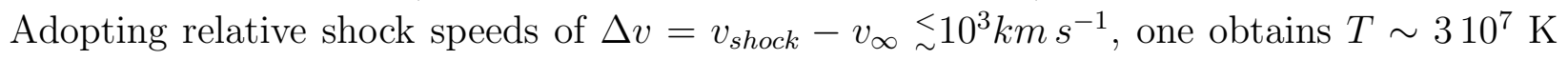
for the shocked gas (e.g. Stevens et al. 1992). Assuming that the cooling time is related to the disappearence of the extra-absorption features at $v>v_{\infty}$ (Fig. 1), $t_{\text {cool }} \sim 10^{5} \mathrm{~s}$, and taking the cooling function for the WR wind (e.g. St-Louis et al. 2005), we estimate the density of the shocked gas as $n \sim 10^{9} \mathrm{~cm}^{-3}$. Hence, we observe shocks coming from the same region populated by blobs: $r=6 \ldots 82 R_{\star}$ corresponds to $n=10^{10} \ldots 10^{8} \mathrm{~cm}^{-3}$ in the wind of WR135 (A. Barniske, priv. comm.). Indeed, taking the flow time $t=R_{\star} / v_{\infty}=20 \mathrm{~min}$ (cf. Koesterke et al. 2001) and assuming that blobs originate at the base of the wind and survive

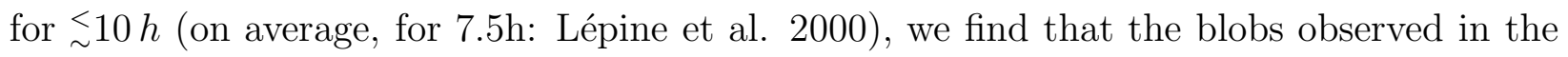
optical populate the region out to $r_{\sim}^{<30} R_{\star}$, in fair agreement with the estimate coming from the UV.

\section{Conclusions}

From our simultaneous detection (both in time and velocity space) of a short-lived (hours) absorption feature in the $v>v_{\infty}$ parts of the emission profiles of lines with a wide range of ionization potentials, including the shock-sensitive OVI doublet, we can conclude that:

(a) in the winds of hot, massive stars the highly blueshifted, $|v|>v_{\infty}$, parts of the $\mathrm{P}$ Cygni profiles may originate in high-speed, shocked regions of the winds;

(b) considering the enormous, $\sim 1 / 4 v_{\infty}$, FWHM of the features (Fig. 2), one can safely assume that a network of shocks, each with an adequate azimuthal extension, may create a flow with highly non-monotonic velocity field, thus resulting in a completely saturated $\mathrm{P}$ Cygni trough - a hypothesis voiced a long time ago by Lucy (1983; with further developments in Puls et al. 1993), but never proved with any certainty; 
(c) the observed shocks may be responsible for the relatively soft X-ray flux from $\mathrm{OB} / \mathrm{WR}$ winds, with relative (to an unperturbed flow) velocities of the shocked gas reaching

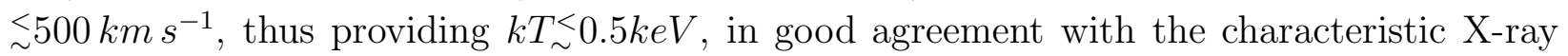
temperatures of single OB stars (Berghöfer et al. 1996);

(d) if shocks and blobs are related, then, considering the propagation of reverse/forward shocks (now the same as blobs) in the wind, we cannot expect them to follow the velocity law of an unperturbed wind. This may explain the seemingly low acceleration of blobs in the WR winds (see the discussion in Koesterke et al. 2001).

We thank Stan Owocki for enlightening discussions of the wide range of issues related to wind instabilities. This work was partially supported by the NASA research grant NAG513716. AFJM and NSL are grateful for financial assistance from NSERC (Canada) and FQRNT (Quebec). We thank the first referee for his constructive criticism which helped to streamline our discussion.

Facilities: FUSE. 


\section{REFERENCES}

Berghöfer, T.W. et al. 1997, A\&A, 322, 167

Berghöfer, T.W., Schmitt, J.H.M.M., \& Cassinelli, J.P. 1996, A\&AS, 118, 481

Cassinelli, J.P., \& Olson, G.L. 1979, ApJ, 229, 304

Cherepashchuk, A.M. et al. 1984, ApJ, 281, 774

Cranmer, S.R., \& Owocki, S.P. 1996, ApJ, 462, 469

Dessart, L. et al. 2000, MNRAS, 315, 407

Donati, J.-F. et al. 2002, MNRAS, 333, 55

Eversberg, T., Lépine, S., \& Moffat, A.F.J. 1998, ApJ, 494, 799

Feldmeier, A., Puls, J., \& Pauldrach, A.W.A. 1997, A\&A, 322, 878

Fullerton, A.W., Massa, D.L., \& Prinja, R.K. 2005, ApJ, 637, 1025

Hamann, W.-R., \& Koesterke, L. A\&A, 1998, 335,1003

Henriksen, R.N. 1994, Ap.Sp.Sc., 221, 25

Hillier, D.J. 1991, A\&A, 247, 455

Hillier, D.J., \& Miller, D.L. 1999, ApJ, 519, 354

Howarth, I.D., \& Prinja, R.K. 1989, ApJS, 69, 527

Kaper, L. et al. 1996, A\&AS, 116, 257

Kaper, L. \& Henrichs, H.F. 1994, Ap\&SpSc, 221, 115

Koesterke, L., Hamann, W.-R., \& Urruitia, T. 2001, A\&A, 379, 224

Lamers, H.J.G.L.M. et al. 1988, ApJ, 325, 342

Lefèvre, L. et al. 2005, MNRAS, 360, 141

Lehner, N. et al. 2003, ApJ, 589, 526

Lépine, S., \& Moffat, A.F.J. 1999, ApJ, 514, 909

Lépine, S. et al. 2000, AJ, 120, 3201 
Lucy, L.B. 1983, ApJ 274, 372

MacFarlane, J.J. et al. 1994, ApJ, 437, 351

Marchenko, S.V. 2003, A Massive Star Odyssey: From Main Sequence to Supernova, K. van der Hucht, A. Herrero, \& E. Csar, San Francisco: ASP, 296

Meynet, G., \& Maeder, A. 2005, A\&A, 429, 581

Moffat, A.F.J. et al. 1988, ApJ, 334, 1038

Moos, H.W. et al. 2000, ApJL, 538, L1

Niedzielski, A., \& Skorzynski, W. 2002, Acta Astron., 52, 81

Oskinova, L. et al. 2003, A\& A, 402, 755

Owocki, S.P. 1994, Ap.Sp.Sc., 221, 3

Puls, J., Owocki, S.P., \& Fullerton, A.W. 1993, A\&A, 279, 457

Repoulst, T., Puls, J., \& Herrero, A. 2004, A\&A, 415, 349

Sahnow, D.J. et al. 2000, ApJL, 538, L7

Savage, B.D., \& Sembach, K.R. 1991, ApJ, 379, 245

Sembach, K.R. et al. 2004, ApJS, 155, 351

St-Louis, N. et al. 1989, A\&A, 226, 249

St-Louis, N. et al. 1995, ApJL, 452, L57

St-Louis, N. et al. 2005, ApJ, 628, 953

Stevens, I., Blondin, J.M., \& Pollock, A.M.T. 1992, ApJ, 386, 265

Wessolowski, U. 1996, MPE Report 253, 75

Willis, A.J. et al. ApJS, 2004, 154, 651

Zsargó, J. et al. 2003, ApJ, 586, 1019 

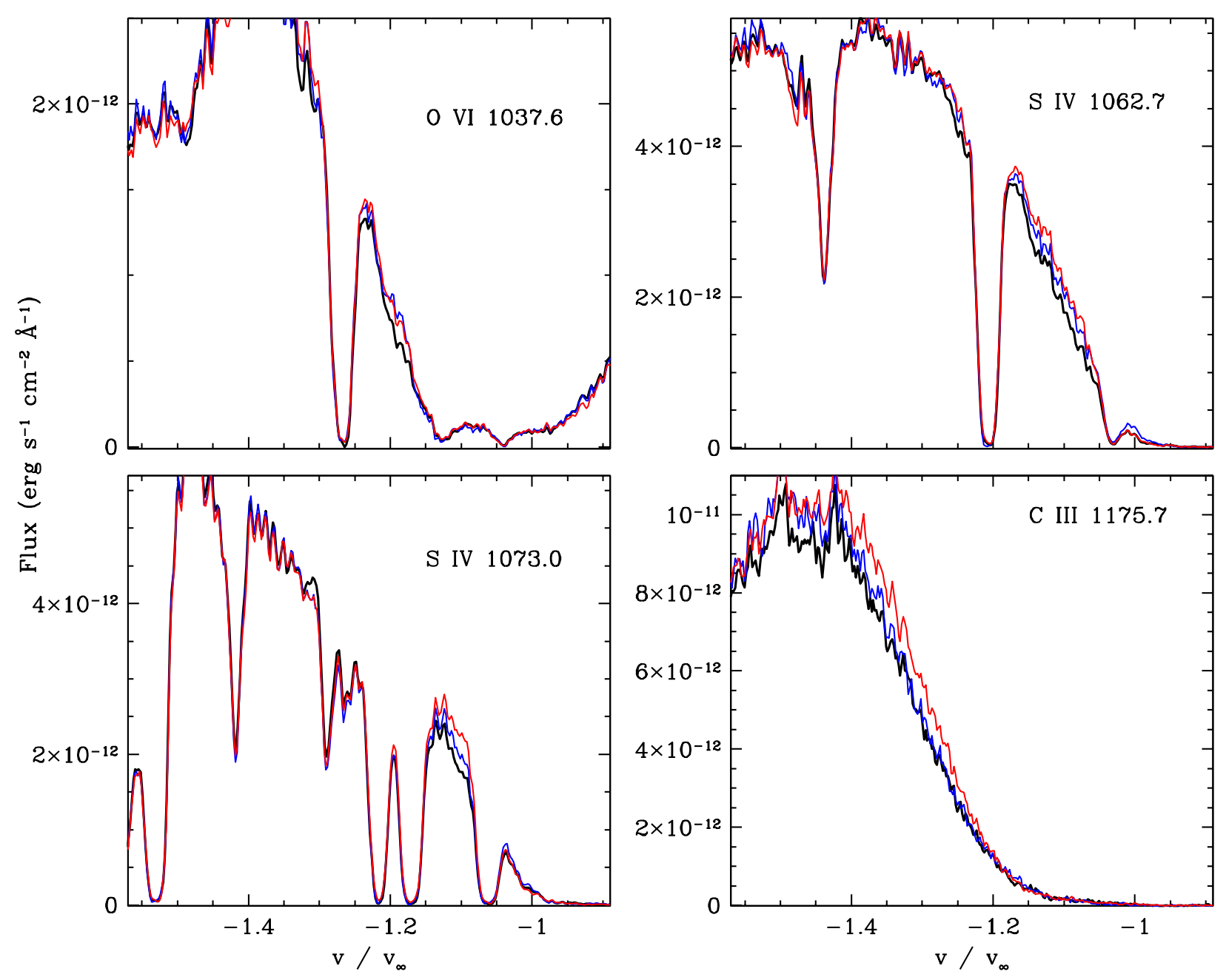

Fig. 1. - Fig. 1. The average profiles of major emission lines grouped as: UT 2:11-5:35 (exp. 1-14, black lines), UT 13:31-15:35 (exp. 30-39, blue lines) and UT 20:34 - 08:11 (next day, exp. 40-59, red lines), i.e. with time progressing from black to red. 


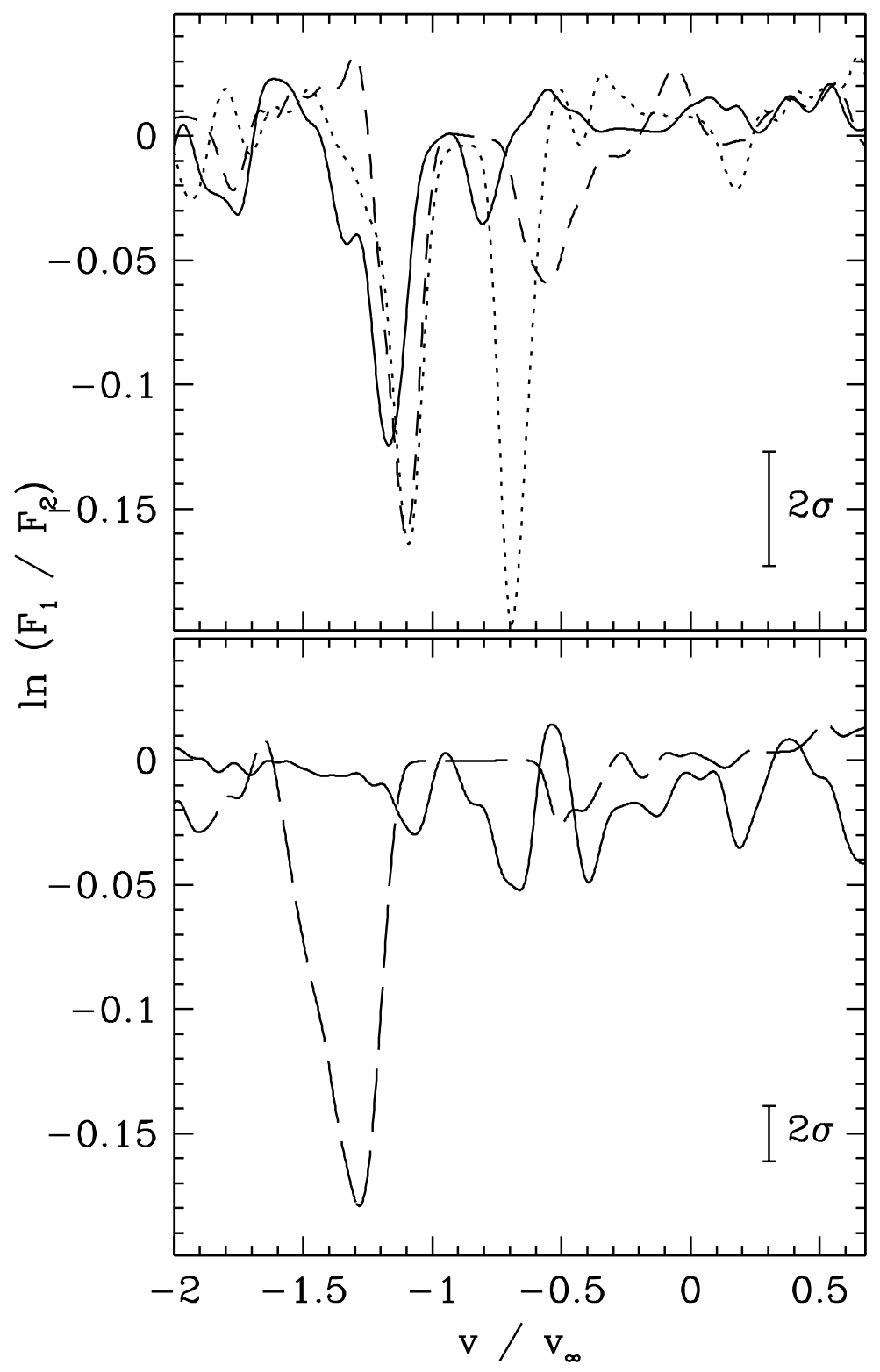

Fig. 2.- Top panel: the differences of apparent optical depths of the variable details in the profiles of OVI (full line), S IV at 1062.7 (dotted line) and S IV at 1073.0 (dashed line). Bottom panel: Si III 1108.4 (full line) and C III 1175.7 (long-dashed line). 